\title{
The technological origins of radical inventions
}

\author{
Wilfred Schoenmakers ${ }^{\mathrm{a}, \mathrm{b}, \mathrm{c}, 1}$, Geert Duysters ${ }^{\mathrm{a}, \mathrm{d}, *}$ \\ a Eindhoven University of Technology, The Netherlands \\ ${ }^{\mathrm{b}}$ Maastricht University, The Netherlands \\ ${ }^{\mathrm{c}}$ Hasselt University, Belgium \\ d Tilburg University, The Netherlands
}

\section{A R T I C L E I N F O}

\section{Article history:}

Received 17 April 2007

Received in revised form 24 March 2010

Accepted 26 May 2010

Available online 1 July 2010

\section{Keywords:}

Radical inventions

Patents

Alliances

Open innovation

Organizational learning

\begin{abstract}
A B S T R A C T
This paper aims to trace down the origins of radical inventions. In spite of many theoretical discussions on the effect of radical inventions, the specific nature of radical inventions has received much less attention in the theoretical and empirical literature. We try to fill that void by an empirical investigation into the specific origins of radical inventions. We explore this issue by a close examination of 157 individual patents, which are selected from a pool of more than 300,000 patents. In contrast to the conventional wisdom that radical inventions are based less on existing knowledge, we find that they are to a higher degree based on existing knowledge than non-radical inventions. A further result that follows from our analysis is that radical inventions are induced by the recombination over more knowledge domains. The combination of knowledge from domains that might usually not be connected seems to deliver more radical inventions.
\end{abstract}

(C) 2010 Elsevier B.V. All rights reserved.

\section{Introduction}

Inventions come in many different forms ranging from incremental or run-of-the-mill inventions, to radical or breakthrough inventions. Most inventions can be characterized as incremental inventions. Incremental inventions consist of minor improvements or plain adjustments to existing products or technology. Their individual impact on the technological system is usually limited. Radical inventions on the other hand are generally considered as being a risky departure away from existing practice (Hage, 1980). Radical inventions exhibit key characteristics that are inherently different from existing products or technologies. They often lie at the hart of changes in technological paradigms (Nelson and Winter, 1982), thereby creating new technological systems and sometimes even new industries. Although incremental inventions might be a principal source of measured productivity growth, without the original radical invention they would not have been possible. Radical inventions are thus considered to be a crucial basis for a sequence of subsequent developments around this original invention (Mokyr, 1990).

In spite of many theoretical discussions on the effect of radical inventions (e.g. Ahuja and Lampert, 2001; Rosenkopf and Nerkar, 2001; Dahlin and Behrens, 2005; Tellis et al., 2009), the specific

\footnotetext{
* Corresponding author at: Eindhoven University of Technology, The Netherlands. E-mail addresses: W.W.M.E.Schoenmakers@tue.nl (W. Schoenmakers), G.M.Duysters@tue.nl (G. Duysters).

1 Tel.: +31 402472170 .
}

nature of radical inventions has so far remained relatively unclear. In fact, large-scale empirical studies into the technological origin of radical inventions are sparse and almost non-existing. Most previous studies on radical inventions have focused on the Schumpeterian size based discussion about the role of small and large firms in the creation of radical inventions and innovations. The empirical results of these studies however remain mixed (Scherer, 1991). Others have focused on organizational aspects influencing the development of radical inventions (for an overview see Chandy and Tellis, 1998). In order to advance theory and practice we will argue that it is critical to understand the specific technological characteristics that influence the development of radical inventions. In contrast to many existing studies we are primarily interested in the technological origins of radical inventions rather than the market success. We therefore depart from the commonly used innovation aspects and focus instead on the invention itself. In particular we would like to contribute to the classic discussion of whether radical inventions are based on completely new knowledge (Poel, 2003) or can be seen as an artefact resulting from the recombination of existing knowledge (Schumpeter, 1939; Fleming, 2001; Nerkar, 2003). A better understanding of the origins of radical inventions might guide organizations in their decisions to either focus on internal development for the creation of new knowledge or to resort to "open innovation" in their search for "neue combinationen" (Schumpeter, 1939). From a societal aspect, more knowledge about the origins of radical inventions is important because of the potential impact of this particular kind of inventions in creating new technological systems or even new industries. 


\section{Theoretical background and hypotheses}

The importance of radical inventions has been demonstrated in many different publications. (e.g. Ahuja and Lampert, 2001; Rosenkopf and Nerkar, 2001; Dahlin and Behrens, 2005). There is a clear consensus among scholars and practitioners that radical inventions are driving forces of technological, industrial and societal change. Whereas the impact of these inventions on the global economy has been described extensively in the literature, much less is known about the particular nature or origins of radical inventions. Apart from a few notable exceptions (e.g. Ahuja and Lampert, 2001) the technical content of a radical invention has not been studied extensively. Instead, existing studies focused on the issue of innovation rather than invention. In a seminal article, Ahuja and Lampert (2001, p. 523) define radical or breakthrough inventions as "those foundational inventions that serve as the basis for many subsequent technical developments". In this definition Ahuja and Lampert address the technical content of an invention. They do not consider the inventions that are radical from a user or market perspective, but instead they focus only on the technological importance of inventions. Second, they define radical inventions as those inventions that serve as a source for many subsequent inventions. Their premise is thus that radical inventions are those inventions whose technical content will be used by many successive inventions (see also Trajtenberg, 1990a; Trajtenberg, 1990b). Dahlin and Behrens (2005), on the other hand, consider technologies to be radical when they are: (1) novel, (2) unique, and (3) have an impact on future technology. The term novel needs some clarification. In this definition they include radical inventions that are constructed of already existing, but beforehand-unconnected knowledge (Hargadon, 2003). In order to be labelled as a radical invention, new knowledge, or the recombination of already existing knowledge must be unique. The last point in the definition of Dahlin and Behrens (2005), concerning the impact of radical inventions on future technology, is in line with the definition given by Ahuja and Lampert (2001). They also consider radical inventions as those inventions with a relatively major impact on future inventions. An invention is thus considered radical if relatively many subsequent inventions build on it. Therefore, impact on subsequent inventions can be seen as a proxy for radicalness. In a similar vein we consider all inventions that serve as an important antecedent for later inventions as radical invention. We thus use the impact of inventions on subsequent inventions as an approximation for the radicalness of an invention. In this study we will discuss their particular nature in retrospect. Hereby we will focus our attention solely on technological inventions.

The discovery of radical inventions is sometimes mystified and glorified. Many people still have an idealised picture of the lone inventor in a laboratory stocked away from the outside world for many years waiting for his/her moment of glory. The lone inventor is rather the exception than the rule (Hargadon, 2003). Although the lone inventor still exists (Dahlin et al., 2004) mostly a team of experts on different fields joins forces in order to develop radical inventions. Another myth is that radical inventions are always based on completely new knowledge (Poel, 2003). In fact, the recombination of existing knowledge is proposed by many scholars to be the ultimate source of novelty (Fleming, 2001; Nerkar, 2003). Even Schumpeter (1939) in the late 1930s considered invention as new combinations or "neue combinationen" (Schumpeter, 1934, pp. 65-66). Nelson and Winter (1982, p. 130) assert “... that invention in the economic system ... consists to a substantial extent of a recombination of conceptual and physical materials that were previously in existence". Even a simple rearrangement of components that are already in use, can, according to Henderson and Clark (1990), be a main cause of destabilisation in key industries. In a similar vein Hargadon and Sutton (1997) have described how firms create novelty by being a technology broker. Fleming states that "... an invention can be defined as either a new combination of components or a new relationship between previously combined components" (Fleming, 2001). According to Hargadon (2003) radical inventions are only rarely based on completely new knowledge. Most of the time radical inventions come from a recombination of already existing knowledge. "When ... connections are made, existing ideas often appear new and creative" (Hargadon and Sutton, 1997, p. 716). Particularly important in this respect is the recombination of beforehand-unconnected knowledge or unconnected knowledge domains (Hargadon, 2003).

However, large-scale empirical evidence is still unavailable and a number of scholars would contend that a radical invention is likely to be based on truly novel knowledge and thus goes beyond simple recombination, irrespective of examples of inventions based on the recombination of existing knowledge or the discovery of a new context for already existing knowledge (Poel, 2003). We believe that radical inventions originate from two basic sources, the recombination of existing knowledge as well as from the creation of truly novel knowledge. Therefore we hypothesize that radical inventions are generally based on existing knowledge.

$\mathbf{H}_{1}$. Radical inventions are equally based on existing knowledge, as non-radical inventions.

As discussed above, radical inventions are for a substantial part dependent on already existing but beforehand-unconnected knowledge. This existing knowledge comes about in two different forms, mature knowledge, and emergent knowledge. The recombination of existing knowledge can therefore be based on either "old" or mature knowledge, or on "new" or emerging knowledge, or on a combination of both. In the literature there is a debate going on about the importance of mature and emergent technologies (Ahuja and Lampert, 2001; Nerkar, 2003). Emerging technologies are technologies that have come to the market only recently, and that are considered to be cutting edge technology (Ahuja and Lampert, 2001). Emerging technologies offer many opportunities for developing new recombinant technologies. Emerging technologies can offer firms valuable new components that facilitate the development of radical inventions (Ahuja and Lampert, 2001). Firms, however often lack the deep understanding of emerging technologies, which is needed to develop radical inventions. Firms that tend to rely on emerging technologies often have difficulties in understanding the real properties of this knowledge and therefore can only contribute in a limited way in terms of developing future technologies (Nerkar, 2003). In contrast, mature technologies are well comprehended and have been tested and used in many different settings. They "are usually well understood and offer greater reliability relative to more recently developed and less tested" technologies (Ahuja and Lampert, 2001, p. 527). Firms, and especially incumbent firms, will prefer mature technologies to nascent technologies. They are more familiar with them, and they are more aware of the specific properties of the technologies. The outcomes of emerging technologies are much more uncertain. This is also related to the concept of absorptive capacity as introduced by Cohen and Levinthal (1990). Firms invest in R\&D and as a result build up absorptive capacity in their organization. Absorptive capacity is generally path dependent and in line with a firms' current research. With emergent technologies, firms will thus, overall, face more difficulties in absorbing them. Firms that focus on the use of existing technologies may benefit from their high degree of absorptive capacity and are therefore often able to speed up the innovation process.

Firms that concentrate on emerging technologies might suffer from experimentation costs and limited output. Dealing with emerging technologies is often problematic. It often takes a long time to discover the specific characteristics of the technology and 
many of the emerging technologies turn out to be less viable then previously expected. Emerging techniques could also ask for different routines, which would require existing employees to change their current routines; routines the employees have been familiar with for a long time, and who are subsequently difficult to change (Nelson and Winter, 1982). Emerging technologies thus, on the one hand pose many opportunities but on the other hand also pose many considerable difficulties that are not easy to cope with in this particular stage of development. In spite of the difficulties that emerging technologies present, we still expect that firms will need emergent knowledge to produce radical inventions. Mature technologies are important, but there is an increasing consensus that emergent technologies are also very important, especially for radical inventions. We would thus expect that radical inventions are, as compared to non-radical inventions, to a higher degree based on emergent technologies.

Our second hypothesis is therefore:

$\mathbf{H}_{2}$. Radical inventions are to a higher extent based on emergent technologies, as compared to non-radical inventions.

In spite of the expected positive relationship between emergent technologies and radical inventions, relying too much on emergent technologies will lead to new knowledge that only has a limited impact on future technologies, while depending too much on mature knowledge might not lead to very innovative ideas or might lead to incremental inventions only (Nerkar, 2003). Mature technologies provide very little opportunities for radical inventions. On the other hand, mature technologies are not always publicly known and are sometimes not used to their full potential at the time of their development and might consequently be forgotten, not because they are not useful, but because at the time of their development they could not be employed. This, for example, has to do with the co-evolution of complementary knowledge, institutions, or standards that are necessary in order to use the new piece of knowledge (Nerkar, 2003). In many cases mature technologies are complemented by other technologies in order to facilitate the rapid development of new inventions. Mature technology is generally well understood as compared to emerging technologies. The combination of mature and emergent technologies could therefore potentially be very beneficial because it allows new combination of different streams of knowledge that might facilitate the development of radical inventions. Furthermore mature knowledge might finally be used to its full potential once complementary technologies become available. We therefore expect that radical inventions are much more based on a combination of mature and emergent technologies.

Our third hypothesis is therefore:

$\mathbf{H}_{3}$. Radical inventions are to a higher degree based on a combination of mature and emergent technologies than non-radical inventions.

Despite the market advantages of combining technologies, firms also tend to search for new knowledge locally, i.e., within the current field of expertise of the firm (Stuart and Podolny, 1996), and within the same geographical confinement (Verspagen and Schoenmakers, 2004). Firms often treasure the convenience of technological and geographic proximity in their search process. They tend to stick to their current structures and routines. As a result, companies often suffer from bounded rationality and are therefore often dealing with only a limited subset of the total knowledge domain. According to Granstrand et al. (1997, p. 13) the technological competencies of large firms depend heavily on their past and are fairly stable. Knowledge is thus "imperfectly shared over time and across people, organizations, and industries" (Hargadon and Sutton, 1997, p. 716). This could potentially lead to the development of "core-rigidities" (Leonard-Barton, 1995) and to the emergence of "competency traps" (Levitt and March, 1988). These traps could well prevent the firm from developing radical inventions. Research by Sorensen and Stuart (2000), for instance, suggests that firms that rely more on their previously developed knowledge deliver more inventions, but these inventions are less relevant.

Research by Granstrand et al. (1997), Patel and Pavitt (1997) and Brusoni et al. (2001) suggests that a firm's technological portfolio typically is larger than its product portfolio. The reason for this is that firms need to search for interesting technologies emerging outside their core technological domain. This broad perspective on technological competencies is thus necessary for firms in order to explore and exploit new technological opportunities (Granstrand et al., 1997). Firms that aim to innovate often need a broader knowledge base in order to do so. This also implies that radical inventions are based on various knowledge domains. Radical inventions not only serve as the basis for many successive inventions (Trajtenberg, $1990 b)$, but can also be expected to build on a larger knowledge base (Rosenkopf and Nerkar, 2001). Differences in terms of the number of knowledge components that make up an invention will appear in all kinds of incremental as well as in radical inventions. A larger knowledge base on the other hand also points at the diversity in the number of different knowledge bases or knowledge domains that constitute an invention. Radical inventions can be expected to draw from a broader knowledge pool than non-radical inventions. If we assume that radical inventions are based on new combinations of already existing knowledge, as discussed before, then this combined knowledge legacy can be expected to come from various, different knowledge domains. In today's world it is very unlikely that radical inventions are based on just one single knowledge domain.

Our fourth hypothesis is therefore:

$\mathbf{H}_{4}$. Radical inventions are based on a relatively large number of knowledge domains, compared to non-radical inventions.

\section{Data}

For our research we will be looking at so-called radical inventions. Inventions are associated with the development of a new idea, whereas innovations refer to the commercialization of this idea (Schumpeter, 1934; Hitt et al., 1993; Ahuja and Lampert, 2001). As discussed we will not be looking at the commercialization of an idea in this paper, but rather at the act of creating an idea. We are particularly interested in how an invention can be a catalyst for the development of subsequent inventions. We especially want to focus on those inventions that can be considered radical or breakthrough. Therefore we focus our attention to those inventions that serve as a basis for many successive inventions.

Patent data is the single most dominant indicator in invention studies. For a patent to be granted it must be novel, non-trivial, and useful. If a patent meets these requirements, a legal title will be created containing information on for instance the name of the inventing firm and also on the technological antecedents of the knowledge, the patent citations. In the European Patent Office(EPO) system, the patent applicant can include citations to prior patents (and prior technological and scientific literature), but ultimately it is the patent examiner from the patent office who determines what citations will be included in a patent (Michel and Bettels, 2001). Patent citations reveal the so-called "prior art" of the newly developed patent. Citations to other patents, the so-called backward citations, indicate on what preceding knowledge the new patent is based. They provide a kind of patent family tree. The citations from other patents to a patent, the so-called forward citations, on the other hand are an indication for the importance of the patent. Patents with higher numbers of forward citations are considered to 
also have a higher economic value for the firm possessing the patent (Trajtenberg, 1990a; Harhoff et al., 1999). Forward citations are also considered to be a good indication for the technological importance of an invention (Dahlin and Behrens, 2005). Firms with more highly cited patents also enjoy economic benefits (Trajtenberg, 1990a) and have on average higher stock market values (Hall et al., 2001).

In the research of Harhoff et al. (1999) it was shown that firms are willing to pay the renewal fees only for important inventions, which leads firms to have only the maximum patent protection for important inventions, leaving less important inventions with a shorter patent protection period. This behavior leads to more citations for important inventions since they have a longer patent-life, but on the other hand they also find that, of the patents with a fullterm patent protection period, the citation frequency rises with the economic value of the invention, as reported by the firm.

In line with the research of Ahuja and Lampert (2001) we will use forward patent citation counts to identify radical inventions, and will consider inventions radical if they serve in a more than average way as the basis for subsequent inventions. Patent citation counts are considered to be good estimators of the technological importance of inventions (Narin et al., 1987; Albert et al., 1991). Highly cited patents are also considered an important indicator for radical inventions (Trajtenberg, 1990a). We will base our definition of radical inventions on Ahuja and Lampert's (2001) definition, as described above. Dahlin and Behrens' (2005) definition is also in line with the definition given by Ahuja and Lampert. Dahlin and Behrens (2005) define inventions as radical if they are (1) novel, (2) unique, and (3) have an impact on future inventions. Since patents are supposed to be novel and non-trivial, covering more or less prerequisites 1 and 2, their definition is the same, in the case of patents, as the one by Ahuja and Lampert (2001). So we are looking for patents with a more than average influence on subsequent patents. We will be using the EPO (European Patent Office) database of patent data as our primary data source.

Patent citations are often referred to as "noisy indicators" of knowledge flows (Jaffe et al., 1998, 2000). The reason being that large parts of patent citations may not be related to a particular knowledge flow due to the fact that patent citations are included not only by the inventor, but as well by the patent attorney of the firm and the patent examiner in the patent office. Recent research has concentrated on the distinction between inventor citations and non-inventor citations (Alcacer and Gittelman, 2006; Criscuolo and Verspagen, 2008). Where Criscuolo and Verspagen propose only to consider inventor citations as knowledge flows, Alcacer and Gittelman conclude that the bias introduced by the examiner citations is not necessarily bad, since both inventor citations and examiner citations might track each other closely. Also, in the EPO system, inventors might make use of knowledge without being aware of the existence of a patent for this piece of knowledge, or without even bothering to include a citation. Inventors also might simply forget to include a citation, or even deliberately not include a citation for strategic reasons. In all these cases a knowledge flow exists but is not visible in the inventor citations. However un-logical these examples might sound in the US Patent and Trademark Office (USPTO) system, in the EPO system they are not. Especially in the EPO system, which we are using for our research, it is the patent examiner of the patent office who is ultimately responsible for including all the patent citations that are necessary, and not the inventor. Together this might also be the reason why Criscuolo and Verspagen's (2008) finding that inventor citations and examiner citations in EPO do not track each other differs from the findings of Alcacer and Gittelman (2006) for USPTO. Also in terms of legal reasons inventor and non-inventor citations in USPTO will track each other more closely than these citations will do in EPO. Furthermore, due to the different requirements of EPO and USPTO, also the examiner included citations in EPO can be expected to be much more related to the prior art of the invention, as would follow from the examples given here before. So in contrast to Criscuolo and Verspagen (2008) we do not feel that in the EPO system it is only the inventor citations that should be considered when looking for knowledge flows. Although we agree with them that, especially when compared to USPTO, inventor citations in EPO very probably do indicate a knowledge flow, in EPO also non-inventor citations might very well be an indication of a knowledge flow. In other words, we cannot exclude the possibility that a non-inventor citation indicates a knowledge flow in the EPO system. In USPTO there is furthermore the problem that applicants include even remotely related citations just to make sure that they do not run any risk of compiling an incomplete list of citations (Michel and Bettels, 2001) which practice introduces "noise" already at the inventor citations. EPO citations can thus be considered less "noisy", for the included citations are less influenced by the fear of legal repercussions (Criscuolo and Verspagen, 2008). Duguet and Macgarvie (2005) finally conclude that patent citation counts are relevant for knowledge flows, although not for all the channels though which firms obtain knowledge. Admitting thus that patent citation are a "noisy" indicator of knowledge flows we still feel confident that they can be used as an indicator of knowledge flows for our purpose, especially since we are using the less "noisy" EPO data. Further, even though we collect our data on the individual patent level, for our analysis we make use of aggregated scores for the two groups of patents that we consider, and as an aggregated variable patent citations are considered to be useful regardless of their individual "noisiness" (Jaffe et al., 2000).

While using the EPO database we might encounter two inherent problems. The first has to do with the difference of the number of patents applied per measuring year. Previous research (see Schoenmakers, 2005) has shown that in EPO data the number of patents applied increases from the start of EPO in 1978 till about 1989. From thereon the number of patents applied stays more or less stable. We cannot use the period where the number of patents applied is not stable, since patents who get applied in a period where there were relatively few other patents applied will have, only because of this reason, less chance of receiving forward citations compared to a patent that is applied in a period with more other patents applied. This is the case, simply because there are more patents that potentially could cite the specific patent. This is especially true since we know that the bulk of forward citations are received within the first four to five years after the initial patent application (Schoenmakers, 2005). Since we do not want the number of forward citations per patent to be dependent on the number of patents applied in a given year but only on the technological importance of the patent, we need to confine our research to that period where the number of patents applied in EPO is more or less equal, which is the period from 1989 till $1998 .^{2}$

A second problem might occur when we compare patents from different periods with each other. Older patents will have a higher chance of receiving forward citations, simply because the period over which the citations are counted is longer compared to younger patents. In order to tackle this problem we counted for every patent the number of forward citations it received up till five years after

\footnotetext{
2 Normalization of the measuring years would also have been possible that would have been the other option to correct for the differences in numbers of patent per year. This would have made it possible to use a longer time period. We choose however to correct this problem by only considering the years with more or less equal numbers of patents. An important reason for our choice was that since the time period that we consider is relatively short we can also expect that other variables, which we cannot control via normalization or otherwise, are more or less constant over the measuring period. We therefore felt that using our approach was the most appropriate choice in this specific context.
} 
its initial application date (usually the filling date of the patent). ${ }^{3}$ This means that for patents applied for in January 1989, we counted the citations up to and including those applied in January 1994, for patents applied in February 1989 we counted the citations until February 1994, etc. Since we can only use the patents between 1989 till 1998, the last year we used patents from is 1993. A similar approach is used by earlier research (Schoenmakers, 2005). Eventually we got a list of 300,119 patents that were applied for in the period from January 1989 till December 1993 with their individual numbers of forward citations.

Since we expect, in line with Ahuja and Lampert (2001) and Dahlin and Behrens (2005), that radical inventions are a rather rare phenomenon, we selected only the most highly cited patents as our group of radical patents. The highest cited radical patent received 54 citations and the least cited 20 citations. We put our cut-off value at 20 citations based on the before mentioned expectation that truly radical inventions will rather be an uncommon occurrence, and we observed that many patents have 19 or less citations, whereas only very few have more than 20 citations. Although this cut-off value of 20 might still seem rather arbitrary, one has to consider the severity of the mistake that we might make. We could either forget to include some of the truly radical inventions or we might include some non-radical inventions in our radical group. In both cases this could only weaken our results, meaning that if we find a difference between radical and non-radical inventions, our results could even have been stronger if we had used a different cut-off point. We therefore feel confident that our cut-off point is not leading us to make a major mistake. Since the mistake of excluding some of the radical inventions from the radical group would altogether lead to the highest chance of making the smallest mistake we choose to be rather conservative with marking inventions as radical. We are therefore convinced that the construction of our group of radical inventions is suitable for our research questions. We ended up with a group of 96 radical patents.

For the construction of the non-radical inventions we randomly selected 96 patents from the group of patents with less than 20 forward citations. For both groups we collected the necessary variables using, besides EPO, Worldscope. We ended up with 74 patents in the radical group and 83 patents in our non-radical group for whom we had sufficient information to perform the analysis.

A small note on the use of patent citations in our research is necessary here. Although we are using patent citations both as a means of assigning patents to either one of the two groups, and as independent variable we feel confident that we can do this. We use the patent's forward citation to be able to assign the patent to one of the two groups and we use the patent's backward citations as dependent variable. So although we use patent citations in both instances the two groups of citations come from two different sources and can therefore be regarded as independent.

Finally, in Appendix A we highlight a few of the patents in the radical group to give the reader some understanding of the type of patents that are in this dataset. Also in this appendix we show the distribution of the radical patents over the different patent classes and over the different measuring years.

\section{Methods}

In order to test if the two groups we are considering, the radical inventions and the non-radical inventions are truly different and to see on what factors they differ we made use of discriminant function analysis. Discriminant function analysis is a statistical technique allowing us to study the difference between two or more

\footnotetext{
${ }^{3}$ We also collected data for a six year citation period but the results remained the same.
}

groups of items with respect to several variables simultaneously. Its primary goal is to distinguish those variables on which groups differ (Tabachnick and Fidell, 1996). Discriminant analysis will aid us in analyzing the differences between groups, and provide us with the weights of the influence of the different individual variables on this difference. Discriminant analysis is thus the appropriate technique for us here, since we want to establish that the two groups differ, and we are especially interested to know on which factors.

Patents applied at the European Patent Office will represent the inventions that we are studying in this research. We thus collect information on the individual patent level, but as discussed before, patents are assigned to two different groups, radical inventions and non-radical inventions, based on the number of forward citations they receive over a specific time period. Our unit of analysis is thus the group of radical and non-radical patents.

Our dependent variable (RAD) is a dummy variable with value zero $(0)$ when the patent is assigned to the non-radical group and one (1) if the patent is in the radical group. This is another important reason why we are using discriminant analysis. Normal regression analysis can only handle a continuous dependent variable, discriminant analysis is on the other hand able to work with a categorical dependent variable as we are using here.

As a first independent variable we use the number of times a patent is citing other patents (COP). Some scholars assert that radical patents are based on completely new knowledge; knowledge that was not available in the market before, while others especially point at the recombination of beforehand-unconnected knowledge as a source of radical inventions. For scholars in favour of the first viewpoint the assumption is, that, if a relatively large amount of citations for a new technology is to scientific literature, than this is an indication of novelty (Carpenter et al., 1981), since the new technology in that case is than not based on already existing technologies, but instead on science itself (Dahlin and Behrens, 2005). Ahuja and Lampert (2001) for instance simply count the number of backward citations and postulate that patents that cite no other patents apparently have no technological antecedents (Ahuja and Lampert, 2001), which would then be an indication for the originality and creativity of the technology (Trajtenberg et al., 1992). Our expectation is however that the discovery of truly novel radical knowledge, in the sense that the knowledge components itself were never before established, is a rather rare phenomenon, occurring only very infrequently. Further in the EPO system it is the patent examiner who is ultimately responsible for the inclusion of "prior art". The chances of an examiner including no, or only a very limited number of backward citations is, already for legal reasons, very small (Michel and Bettels, 2001). Using our variable COP we will be able to test both assumptions.

The second independent variable we use is the mean age of the patents that our studied patents are based upon. This is thus the mean age of the patents that receive the backward citations. From the literature we know that radical patents might be using younger knowledge. Younger knowledge is on the one hand interesting for the opportunities it gives for the development of new knowledge, but is on the other hand more difficult to use since people are not yet quite familiar with the knowledge. Our variable (AGE) is meant to gain us more insight into this phenomenon.

Our third independent variable is the spread of the age of the backward citations (SPREAD). Some studies point to the fact that making use of old and emergent knowledge can produce radical inventions. Knowledge might be developed in a time when this knowledge is not readily usable. Complementary knowledge or techniques might first have to be developed. With our variable SPREAD we can investigate this relationship.

As a fourth independent variable we included the number of different sectors where the knowledge for a new patent comes from. These are thus the sectors where the backward citations of our 
Table 1

Correlations.

\begin{tabular}{lrrrrr}
\hline Correlations & \multicolumn{1}{l}{ COP } & \multicolumn{1}{c}{ AGE } & SPREAD & \multicolumn{1}{c}{ SEC } & \multicolumn{1}{c}{ REV } \\
\hline COP & 1.000 & -.064 & .712 & .448 & .001 \\
AGE & -.064 & 1.000 & -.176 & -.045 & .119 \\
SPREAD & .712 & -.176 & 1.000 & .401 & .087 \\
SEC & .448 & -.045 & .401 & 1.000 & .035 \\
REV & .001 & .119 & .087 & .035 & 1.000 \\
\hline
\end{tabular}

Table 2

Tests of equality of group means.

\begin{tabular}{llrl}
\hline & Wilks' lambda & \multicolumn{1}{l}{ S } & Sig. \\
\hline COP & .844 & 28.585 & .000 \\
AGE & .958 & 6.794 & .010 \\
SPREAD & .936 & 10.533 & .001 \\
SEC & .894 & 18.449 & .000 \\
REV & .980 & 3.195 & .076 \\
\hline
\end{tabular}

studied patents come from. These sectors again, are based on the International Patent Classification (IPC) codes of the cited patents. Hereby we divided the patents up into the different IPC classes, the second hierarchical level of the classification (e.g. A01; B01; B04) In the literature we find many indications pointing to a relationship between diversity of knowledge and radicalness of the invention. Our variable (SEC) is used to test this relationship.

As a control variable we use the revenues of the firms that applied for the individual patents (REV). This helps to eliminate the influence that the size of the firms might have on the results.

The function that we are studying then becomes:

$\mathrm{RAD}_{k m}=f\left(\mathrm{COP}_{k m}, \mathrm{AGE}_{k m}, \mathrm{SPREAD}_{k m}, \mathrm{SEC}_{k m}, \mathrm{REV}_{k m}\right)$

where $k m=$ case $m$ in group $k$.

\section{Results}

Based on the function discussed above we aim to investigate which variables are distinguishing between the two groups under investigation. We started with investigating the correlations between our independent variables.

It turned out that there are no problems with correlation related issues. All correlations are well within the acceptable range as can be seen from Table 1 . The only somewhat higher correlation is between the two variables SPREAD and COP. It is logical that these two variables have a higher correlation since the chance that SPREAD is high increases when more patents are cited, thus when COP increase. But although the correlation for these two variables is the highest of all the correlations with a value of 0.712 , this is still within the acceptable range.

Next we continued our examination by looking at the Wilks' lambda and $F$ values of the different variables, see Table 2 .

Wilks' lambda is a multivariate measure of the group differences over several variables, it is concerned with the ratio between the within group variance and the overall variance. A ratio near 1 is an indication of equality of group means, whereas a lower ratio is an indication of a larger difference between the various group means. The $F$-ratios are calculated for each variable in order to test the hypothesis that all group means are equal. From Table 2 we see that although the Wilks' lambdas are high, the F values are high too. The results of our discriminant analysis further show that except for our control variable REV, all other variables are highly significant at the $1 \%$ error level. This thus supports a strong rejection of the hypothesis that all group means are equal. For all our independent variable we can therefore reject the hypothesis that the group means are equal.
Table 3

Eigenvalue and Wilks' lambda.

\begin{tabular}{clllll}
\hline Function & Eigenvalue & \% of variance & Cumulative \% & Canonical correlation \\
\hline \multirow{2}{*}{ Test of function } & 100.0 & 100.0 & .472 & \\
\hline & Wilks' lambda & Chi-square & df & Sig. \\
\hline & .777 & 38.440 & 5 & .000 \\
\hline
\end{tabular}

Table 4

Group statistics.

\begin{tabular}{lll}
\hline & Mean & Std. deviation \\
\hline COP non-radical & 1.2651 & 1.29807 \\
AGE non-radical & 138.5635 & 27.95243 \\
SPREAD non-radical & 12.2651 & 20.51952 \\
SEC non-radical & .7470 & .76259 \\
REV non-radical & $24,180,624$ & $24,706,586$ \\
COP radical & 2.5541 & 1.71330 \\
AGE radical & 149.6747 & 25.13730 \\
SPREAD radical & 26.0541 & 32.03974 \\
SEC radical & 1.1757 & .41737 \\
REV radical & $32,492,395$ & $33,326,894$ \\
\hline
\end{tabular}

Looking at Table 3 we can evaluate the discriminatory power of the complete set of variables. This is an indication of the 'goodness of fit' of the discriminant function.

First we look at the eigenvalue, which is a measure for the relationship of the sum of squares of the between group and the within group. Higher eigenvalues indicate a more discriminating function. The discriminating power of the discriminant function is, according to the eigenvalue not high. We then look at the canonical correlation, which represents the proportion of the total variance that is explained by the two different groups, and which is in this case more important than the eigenvalue since we only have two groups. This coefficient is a measure of association summarizing the degree of relatedness between the two groups and the discriminant function. A value of zero means no relationship at all, while large positive numbers represent increasing degrees of association, where 1.0 is the maximum (Klecka, 1980). A value of .472 for the canonical correlation indicates that the discriminating power of our discriminant function is medium.

Next the test of function column tests the hypothesis that the mean of the function listed is equal across groups. The Wilks' lambda here is the proportion of the total variance in the discriminant scores not explained by differences among groups. The value of Wilks' lambda of .777 indicates that the group means do differ. Next, the chi-square value of 38.440 and a significance level of .000 also imply that the mean scores of the different variables for the two researched groups do differ significantly. The null-hypothesis that the two group means are equal can therefore be rejected.

If we look at Table 4, which reports the mean and standard deviation for the different variables for both groups, and when we combine these findings with the results we got from Table 2 we can review our main hypotheses. Looking at the first variable (COP) we see that the group of radical patents cites more patents than the group of non-radical patents, 2.5541 compared to 1.2651 . This variable is also significant (see Table 2). Our first hypothesis is thus rejected. Radical patents are apparently even more reliant on the recombination of already existing knowledge than non-radical patents.

We additionally looked at the percentage of citations to patents from other firms, compared to the percentage of citations of their own patents (self citations). It turned out that for both groups; about $75 \%$ of the citations are to patents from other firms. This implies that for new knowledge construction firms are very much relying on outside knowledge. 
The variable AGE is higher for the group of radical patents compared to the group of non-radical patents. Since we measure the age of a patent in months starting at 1 at the start of EPO and counting up, a higher value for AGE means that the group of radical patents is actually based, on average, on younger patents than the group of non-radical patents. The variable AGE is also significant. Emerging technologies apparently play an important role in the development of radical patents. This clearly confirms our hypothesis 2 .

For our variable SPREAD we see that this variable is larger for radical patents than for non-radical patents. This third independent variable is also significant. Radical patents are thus based on a broader time spectrum of patents. This result is in line with our hypothesis 3.

For our variable SEC we find that the group of radical patents cites more sectors as the group of non-radical patents, 1.1757 compared to 7470 . According to Table 2 also this variable is significant. Hypothesis 4 is therefore accepted. Radical patents are based on more sectors, and thus based on more knowledge domains.

Our control variable indicates that larger firms have more radical patents, but this variable is only significant at the $10 \%$ level.

\section{Discussion and conclusions}

In this study we investigated the origin of radical inventions, and compared this with that of non-radical inventions. We based our results on a sample of 157 individual patents selected from a pool of more than 300,000 patents.

In contrast to the conventional wisdom that radical inventions are based less on existing knowledge, we find that they are to a higher degree based on existing knowledge than non-radical inventions. For radical inventions already existing knowledge seems of paramount importance. Radical inventions are also to a higher degree based on emergent technologies, and especially on a combination of mature and emergent technologies than non-radical inventions. As discussed before, the use of emergent technologies might be more difficult for companies because of the newness of the technology. This finding might also be very important in the light of the rise of open innovation. Based on our results we can see that open innovation might be an important contributor in the development of radical inventions. Alliances and open innovation systems might facilitate the diffusion of knowledge over firms and within firms much better, adding to the chances of recombining mature and emergent knowledge. This is something that certainly needs to be investigated further in future research.

It also shows the importance of speed in understanding emergent technologies. Firms that are quick in understanding the possibilities that emergent technologies posses, and that therefore are able to combine this knowledge with mature and wellunderstood knowledge, might be better at delivering radical inventions. The results also show that caution is needed with discarding mature technologies too quickly; they might be useful in a later, different setting.

A further result that follows from our analysis is that radical inventions are induced by the recombination over more knowledge domains. The combination of knowledge from domains that might usually not be connected seems to deliver more radical inventions. Also this might be enhanced by alliances and an open innovation system. By means of alliances firms will be able to tap more easily into knowledge that otherwise would be inaccessible for them.

Our results also have bearing on the internal management of firm knowledge. It shows the need for more coordination of the knowledge within the firm, and more internal openness. Different divisions might possess knowledge that, when put together, could potentially deliver a radical invention. It might be beneficial for firms to have multidisciplinary teams of people from all over the firm to work together and scan the internal as well as the external environment for possible beneficiary combinations of knowledge. Technology brokers might here also convey an important influence.

In spite of these important contributions there are also some limitations that have to be acknowledged. First, although statistically sufficient, we need to consider that especially for our non-radical patent group, even though we selected them at random, there is a chance that they do not fully represent the non-radical patent group. Also the decision to have our cut-off point for radical inventions at 20 is subject to discussion, although, as discussed before, we think that we ensured that we made the correct assessment. The discriminating power of our discriminant function is medium, although the individual factors show to be significant and discriminating between the two researched groups, and also Wilks' lambda indicates that the group means do differ.

We must also acknowledge that, although our radical patents come from many diverse patent classes, some patent classes are represented more than others (see Appendix A). This does certainly justify future research into this matter. Also as a complement to our current research, for future research it would be interesting to investigate some of our 157 inventions deeper, maybe by using interviews at the involved companies. This could also further strengthen our current results.

We must acknowledge that the technological origin of radical inventions explains only part of the emergence of radical inventions. Other authors have pointed, among others, at the role of the Government, organizational characteristics, the beneficial effects of collaboration with university institutes and the importance of culture (Tellis et al., 2009)

Given these limitations, based on the results of our discriminant function analysis we still feel comfortable with our results and can come to some very interesting conclusions concerning radical inventions. Our research thus hints on the importance of alliances and an open innovation system for the development of radical inventions. It can also help firms to focus their attention to the most important factors leading to the development of a radical invention. Not just the new scientific knowledge but also the recombination of already existing knowledge, mature and emergent, from different knowledge domains is vital. The most important lesson that follows from these results might be that there could be many more radical inventions out there, waiting to be discovered by researchers that are able to recombine previously unconnected knowledge. Researchers that are able to look over their own narrow research borders, both in time and in a technological and a geographical sense, and that are willing to use outside knowledge and share inside knowledge might be the masters of radical invention.

\section{Acknowledgements}

We would like to thank Wim Vanhaverbeke, Christian Kerckhoffs and the two anonymous referees for their valuable comments on earlier versions of our paper.

\section{Appendix A.}

In order to provide some background information about the patents that are in our radical patents group we here discuss some of them. The information is obtained from the EPO internet page esp@cenet.com. For more elaborate information on the specific patents please contact this website. The information given here is intended to only give some background information about some of the patents. 
Table 5

Number of radical patents per Main IPC Classification in our sample.

\begin{tabular}{|c|c|c|c|c|c|c|c|c|c|c|c|c|c|c|c|}
\hline A61 & B41 & B65 & $\mathrm{CO} 7$ & $\mathrm{CO8}$ & C09 & C10 & C11 & C12 & F01 & F02 & G03 & G06 & G07 & G11 & H04 \\
\hline 4 & 3 & 1 & 31 & 4 & 1 & 2 & 1 & 4 & 2 & 1 & 2 & 1 & 1 & 3 & 13 \\
\hline
\end{tabular}

One of the patents in our radical patent group is patent EP0324377 with the title: Angiotensin II receptor blocking imidazoles and combinations thereof with diuretics and NSAIDs.

The invention encompasses a pharmaceutical composition comprising a diuretic or a non-steroidal anti-inflammatory drug, a pharmaceutical suitable carrier and an antihypertensive and processes for production of these compositions.

This invention relates to novel substituted imidazoles, and processes for their preparation. The invention also relates to pharmaceutical compositions containing the novel imidazoles and pharmaceutical methods using them, alone and in conjunction with other drugs, especially diuretics and non-steroidal antiinflammatory drugs (NSAIDs)

Another patent in our radical patent group is patent EP0342060 with the title: Image processing apparatus.

The present invention relates to an image processing apparatus for processing an input image and recording the image on a recording medium, and more particularly to an image processing apparatus which is suitably applied to a color copying apparatus that is capable of effecting the color copying of an image of an original.

A third example is patent EP0369707: "Arrayed disk drive system and method". This invention deals with a memory system for a computer. More specifically, the invention relates to arranging a plurality of disk drives in an array and causing the array to have flexibility in configuration. Among the aspects of the array that are flexible are the logical configuration of the disk drives, the level of redundancy available, and the allocation of spare disk drives.

A fourth patent is EP0622002: Digital video signal processing apparatus and method. This invention relates to an apparatus and method for processing a variable-rate coded signal prior to recording so that high-speed searching can be carried out on a recording medium on which the processed signal is recorded. The invention also relates to an apparatus and method for performing a highspeed search on a medium on which a processed variable-rate coded signal is recorded. Finally, the invention relates to a recording of the processed variable-rate coded signal.

A fifth and final example of a patent from the radical patent group concerns EP0540290: Expandable stents and method for making same.

This invention relates to expandable endoprosthesis devices, generally called stents, which are adapted to be implanted into a patient's body lumen, such as blood vessel, to maintain the patency thereof. These devices are very useful in the treatment of atherosclerotic stenosis in blood vessels. Stents are generally tubular shaped devices which function to hold open a segment of a blood vessel or other anatomical lumen. They are particularly suitable for use to support and hold back a dissected arterial lining which can occlude the fluid passageway there through.

In order to provide further insight into the total pool of 74 radical patents that we have used for our analysis we included two tables, Tables 5 and 6 with extra information on these patents. Table 5

\section{Table 6}

Number of radical patents per year in our sample.

\begin{tabular}{lllll}
\hline 1989 & 1990 & 1991 & 1992 & 1993 \\
\hline 15 & 21 & 10 & 15 & 13 \\
\hline
\end{tabular}

Table 7

Explanation of the codes used. Information obtained from: http://www.wipo.int/ classifications/ipc/.

\begin{tabular}{|c|c|}
\hline $\begin{array}{l}\text { Section A } \\
\text { Human necessities }\end{array}$ & $\begin{array}{l}\text { A61 Medical or veterinary science; } \\
\text { hygiene }\end{array}$ \\
\hline $\begin{array}{l}\text { Section B } \\
\text { Performing operations; transporting }\end{array}$ & $\begin{array}{l}\text { B41 Printing; lining machines; } \\
\text { typewriters; stamps } \\
\text { B65 Conveying; packing; storing; } \\
\text { handling thin or filamentary material }\end{array}$ \\
\hline $\begin{array}{l}\text { Section C } \\
\text { Chemistry; metallurgy }\end{array}$ & $\begin{array}{l}\text { C07 Organic chemistry } \\
\text { C08 Organic macromolecular } \\
\text { compounds; their preparation or } \\
\text { chemical working-up; compositions } \\
\text { based thereon } \\
\text { C09 Dyes; paints; polishes; natural } \\
\text { resins; adhesives; compositions not } \\
\text { otherwise provided for; applications of } \\
\text { materials not otherwise provided for } \\
\text { C10 Petroleum, gas or coke industries; } \\
\text { technical gases containing carbon } \\
\text { monoxide; fuels; lubricants; peat } \\
\text { C11 Animal or vegetable oils, fats, fatty } \\
\text { substances or waxes; fatty acids } \\
\text { therefrom; detergents; candles } \\
\text { C12 Biochemistry; beer; spirits; wine; } \\
\text { vinegar; microbiology; enzymology; } \\
\text { mutation or genetic engineering }\end{array}$ \\
\hline $\begin{array}{l}\text { Section F } \\
\text { Mechanical engineering; lighting; } \\
\text { heating; weapons; blasting }\end{array}$ & $\begin{array}{l}\text { F01 Machines or engines in general; } \\
\text { engine plants in general; steam } \\
\text { engines } \\
\text { F02 Combustion engines; hot-gas or } \\
\text { combustion-product engine plants }\end{array}$ \\
\hline $\begin{array}{l}\text { Section G } \\
\text { Physics }\end{array}$ & $\begin{array}{l}\text { G03 Photography; cinematography; } \\
\text { analogous techniques using waves } \\
\text { other than optical waves; } \\
\text { electrography; holography } \\
\text { G06 Computing; calculating; counting } \\
\text { G07 Checking-devices } \\
\text { G11 Information storage }\end{array}$ \\
\hline $\begin{array}{l}\text { Section } \mathrm{H} \\
\text { Electricity }\end{array}$ & H04 Electric communication technique \\
\hline
\end{tabular}

shows the number of radical patents per Main IPC Classification. ${ }^{4}$ We can see that radical patents can be found in almost all of the IPC sections except for the sections D and E, respectively, Textiles and Paper, and Fixed constructions. Most radical patents in our sample come from section $C$ with 43 patents, followed at some distance by section $\mathrm{H}$ with 13 patents and section $\mathrm{G}$ with 7 patents. The rest of the sections are only represented by three to four patents in our sample. Section $C$ is also represented by six classes in our sample, followed by section $G$ with four classes. The other sections only have patents in one or two classes.

Patent class C07, Organic chemistry, is the class with the highest number of radical patents in our sample, again followed by patent class H04 Electric communication technique. So while there is a concentration of patents from the sections $\mathrm{C}$ and $\mathrm{H}$ and from the classes $\mathrm{C} 07$ and $\mathrm{H} 04$, the patents in our radical patent group apparently come from all sections, except the obvious exemptions. The radical patents are also coming from a very diverse set of patent classes that are not really close to each other. Because of this spread

\footnotetext{
${ }^{4}$ For an explanation of the IPC codes please see Table 7 (further information can be obtained from http://www.wipo.int/classifications/ipc/).
} 
of our radical patents over so many different patent classes, and given the different patenting behaviors in each patent class, we feel confided that our results are not only applicable to patent class $\mathrm{CO} 7$ and $\mathrm{HO} 4$ but can be generalized to the other patent classes as well. Of course future research might be needed to make stronger statements about the generalizability regarding the data.

Also if we look at Table 6 we can see that the radical patents in our sample are more or less evenly distributed over the measuring years. Especially given the small number of patents we are working with, the differences per measuring year are rather small, and acceptable in our opinion.

\section{References}

Ahuja, G., Lampert, C.M., 2001. Entrepreneurship in large corporations: a longitudinal study of how established firms create breakthrough inventions. Strategic Management Journal 22, 521-543.

Albert, M.B., Avery, D., Narin, F., McAllister, P., 1991. Direct validation of citation counts as indicators of industrially important patents. Research Policy 20 (3), 251-259.

Alcacer, J., Gittelman, M., 2006. Patent citations as a measure of knowledge flows: the influence of examiner citations. The Review of Economics and Statistics 88 (4), 774-779.

Brusoni, S., Prencipe, A., Pavitt, K., 2001. Knowledge specialization, organizational coupling, and the boundaries of the firm: Why do firms know more than they make? Administrative Science Quarterly 46 (4), 597-621.

Carpenter, M.P., Narin, F., Wolf, P., 1981. Citation rates to technologically important patents. World Patent Information 3 (4), 160-163.

Chandy, R.K., Tellis, G., 1998. Organizing for radical product innovation: the overlooked role of willingness to cannibalize. Journal of Marketing Research 35 (4), 474.

Cohen, W.M., Levinthal, D.A., 1990. Absorptive capacity: a new perspective on learning and invention. Administrative Science Quarterly 35, 128-152.

Criscuolo, P., Verspagen, B., 2008. Does it matter where patent citations come from? Inventor vs. examiner citations in European patents. Research Policy 37, 1892-1908.

Dahlin, K., Taylor, M., Fichman, M., 2004. Today's Edisons or weekend hobbyists: technical merit and success of inventions by independent inventors. Research Policy 33 (8), 1167-1183.

Dahlin, K.B, Behrens, D.M., 2005. When is an invention really radical? Defining and measuring technological radicalness. Research Policy 34 (5), 717-737.

Duguet, E., Macgarvie, M., 2005. How well do patent citations measure flows of technology? Evidence from French innovation surveys. Economics of Innovation and New Technology 14 (5), 375-393.

Fleming, L., 2001. Recombinant uncertainty in technological search. Management Science 47 (1), 117-132.

Granstrand, O., Patel, P., Pavitt, K., 1997. Multi-technology corporations: why they have "distributed" rather than "distinctive" core competencies. California Management Review 39 (4), 8-25.

Hage, J., 1980. Theories of Organization. Wiley Interscience, New York.

Hall, B.H., Jaffe, A., Trajtenberg, M., 2001. Market value and patent citations: a first look. Berkeley NBER Working Paper.

Hargadon, A., 2003. How Breakthroughs Happen. The Surprising Truth About How Companies Innovate. Harvard Business School Press, Boston, MA.

Hargadon, A., Sutton, R.I., 1997. Technology brokering and innovation in a product development firm. Administrative Science Quarterly 42, 716-749.

Harhoff, D., Narin, F., Scherer, M., Vopel, K., 1999. Citation frequency and the value of patented inventions. Review of Economics and Statistics 81 (3), 511-515.
Henderson, R.M., Clark, K.B., 1990. Architectural innovation: the reconfiguration of existing product technologies and the failure of established firms. Administrative Science Quarterly 35 (1), 9-30.

Hitt, M.A., Hoskisson, R.E., Nixon, R.D., 1993. A midrange theory of interfunctional integration, its antecedents and outcomes. Journal of Engineering and Technology $10(1,2), 161-185$.

Jaffe, A.B., Forgarty, M.S., Banks, B.A., 1998. Evidence from patents and patent citations on the impact of NASA and other federal labs on commercial innovation. Journal of Industrial Economics 46, 183-205.

Jaffe, A.B., Trajtenberg, M., Forgarty, M.S., 2000. Knowledge spillovers and patent citations: evidence from a survey of inventors. American Economic Review 90 (2), 215-218.

Klecka, W.R., 1980. Discriminant Analysis. Sage Publications Inc., Newbury Park, CA. Leonard-Barton, D., 1995. Wellsprings of Knowledge. Harvard Business School Press, Boston.

Levitt, B., March, J.R., 1988. Organizational learning. Annual Review of Sociology 14, 319-340.

Michel, J., Bettels, B., 2001. Patent citation analysis. A closer look at the basic input data from patent search reports. Scientometrics 51 (1), 185-201.

Mokyr, J., 1990. Punctuated equilibria and technological progress. American Economic Review 80 (2), 350-354.

Narin, F., Noma, E., Pery, R., 1987. Patents as indicators of corporate technological strength. Research Policy 16 (2-4), 143-155.

Nelson, R., Winter, S., 1982. An Evolutionary Theory of Economic Change. Harvard University Press, Cambridge.

Nerkar, A., 2003. Old is gold? The value of temporal exploration in the creation of new knowledge. Management Science 49 (2), 211-229.

Patel, P., Pavitt, K., 1997. The technological competencies of the world's largest firms: complex and path-dependent, but not much variety. Research Policy 26 (2), $141-156$.

Poel, I.v.d., 2003. The transformation of technological regimes. Research Policy 32 (1), 49-68.

Rosenkopf, L, Nerkar, A., 2001. Beyond local search: boundary-spanning, exploration, and impact in the optical disk industry. Strategic Management Journal $22(4)$, 287-306.

Scherer, F.M., 1991. Changing perspectives on the firm size problem. In: Acs, Z.J., Audretsch, D.B. (Eds.), Innovation and Technological Change: An International Comparison. Harvester Wheatsheaf, New York, NY.

Schoenmakers, W., 2005. Knowledge Flows Between Multinational Companies. A Patent Data Analysis. Maastricht University Press, Maastricht.

Schumpeter, J., 1939. Business Cycles: A Theoretical, Historical and Statistical Analysis of the Capitalist Process. McGraw-Hill, New York.

Schumpeter, J.A., 1934. The Theory of Economic Development. Harvard University Press, Cambridge.

Sorensen, J.B., Stuart, T.E., 2000. Aging, obsolescence, and organizational invention. Administrative Science Quarterly 45 (1), 81-112.

Stuart, T.E., Podolny, J.M., 1996. Local search and the evolution of technological capabilities. Strategic Management Journal 17 (summer special issue), 21-38.

Tabachnick, B.G., Fidell, L.S., 1996. Using Multivariate Statistics, 3rd edition. Harper Collins College Publishers, Northridge, USA.

Tellis, G.J., Prabhup, J.C., Chandy, R.K., 2009. Radical innovation across nations: the preeminence of corporate culture. Journal of Marketing 73 (1).

Trajtenberg, M., 1990a. A penny for your quotes: patent citations and the value of inventions. Rand Journal of Economics 21 (1), 172-187.

Trajtenberg, M., 1990b. Economic Analysis of Product Innovation: The Case of CT Scanners. Harvard Business School Press, Cambridge, MA.

Trajtenberg, M., Henderson, R., Jaffe, A., 1992. Ivory Tower Versus Corporate Lab: An Empirical Study of Basic Research and Appropriability. NBER Working Paper Series.

Verspagen, B., Schoenmakers, W., 2004. The spatial dimension of patenting by multinational firms in Europe. Journal of Economic Geography 4 (1), 23-42. 